\title{
Erratum: Two-dimensional magneto-optical trap as a source for cold strontium atoms [Phys. Rev. A 96, 053415 (2017)]
}

Ingo Nosske, Luc Couturier, Fachao Hu, Canzhu Tan, Chang Qiao, Jan Blume,

Y. H. Jiang, Peng Chen, and Matthias Weidemüller

(Received 15 February 2018; published 1 March 2018)

DOI: 10.1103/PhysRevA.97.039901

Due to a calibration error of the fluorescence detection, atom numbers and corresponding atom fluxes reported in our article have to be multiplied by a constant factor of 1.6. In particular, this concerns Figs. 3 and 4 as well as Table I, which are reprinted below with the corrected values.

When discussing the results of Figs. 3 and 4 in the text, the atom fluxes also have to be corrected:

(1) Page 5, first paragraph: $1.4 \times 10^{9}$ atoms $/ \mathrm{s}$ instead of $9 \times 10^{8}$ atoms $/ \mathrm{s}$ and $1.0 \times 10^{9}$ atoms $/ \mathrm{s}$ instead of $6 \times 10^{8}$ atoms $/ \mathrm{s}$.

(2) Page 6, second paragraph: $1.4 \times 10^{9} \mathrm{~s}^{-1}$ instead of $9 \times 10^{8} \mathrm{~s}^{-1}$.

The conclusions of our paper are not affected by this correction.

TABLE I. Overview of loading rates of all the stable isotopes of strontium (using flux enhancement of the 2D-MOT by the decreasing field Zeeman slower beam for this set of measurements). $\Delta f$ is the relative frequency shift to ${ }^{88} \mathrm{Sr}$ for the singlet cooling transition. $L_{\text {rel }}$ shows the relative loading rate scaled to the natural abundance.

\begin{tabular}{llcccc}
\hline \hline Isotope & Statistics & Abundance & $\Delta f(\mathrm{MHz})$ & $L\left(10^{6}\right.$ atoms $\left./ \mathrm{s}\right)$ & \multicolumn{1}{c}{$L_{\text {rel }}$} \\
\hline${ }^{88} \mathrm{Sr}$ & Bosonic & $82.58 \%$ & 0 & $480(18)$ & $35(2)$ \\
${ }^{87} \mathrm{Sr}$ & Fermionic & $7.00 \%$ & -51.9 & $61(2)$ & $0.87(6)$ \\
${ }^{86} \mathrm{Sr}$ & Bosonic & $9.86 \%$ & -124.8 & $3.5(8)$ & $1.06(5)$ \\
${ }^{84} \mathrm{Sr}$ & Bosonic & $0.56 \%$ & -270.8 & $1.0(3)$ \\
\hline \hline
\end{tabular}


(a)

a)

(b)

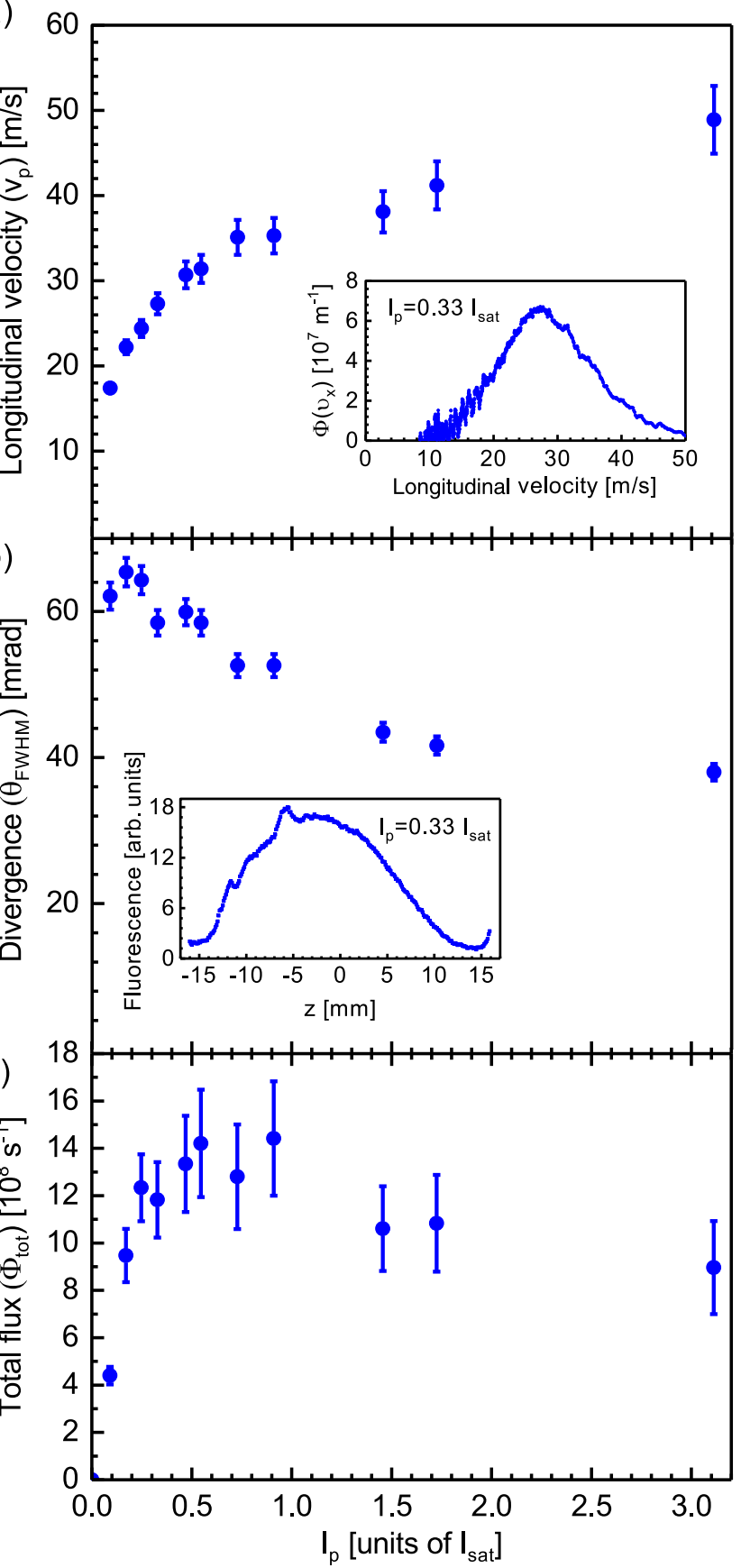

FIG. 3. The most probable longitudinal velocity, divergence, and the total flux of the atomic beam versus the pushing beam light intensity, respectively. The insets in (a) and (b) show the longitudinal velocity distribution and the transverse distribution of the atomic beam at $I_{\mathrm{p}}=0.33 I_{\mathrm{sat}}$. The field gradient is chosen to be $50 \mathrm{G} / \mathrm{cm}$, the oven temperature is $465^{\circ} \mathrm{C}$, and Zeeman slower loading was applied. (a)

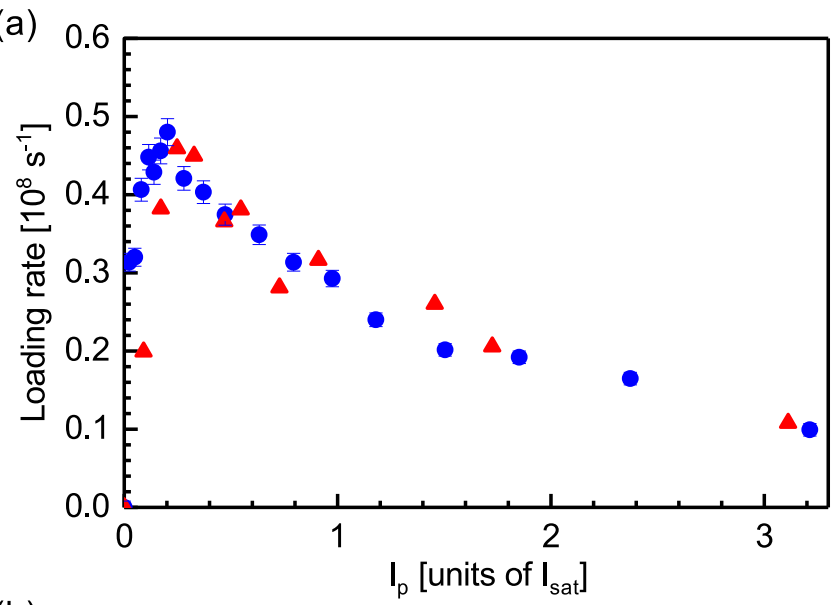

(b)

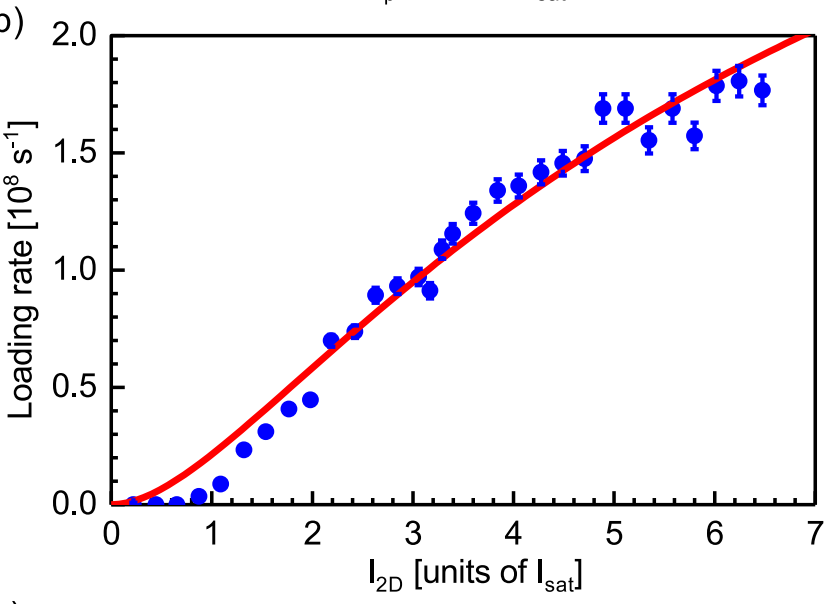

(c)

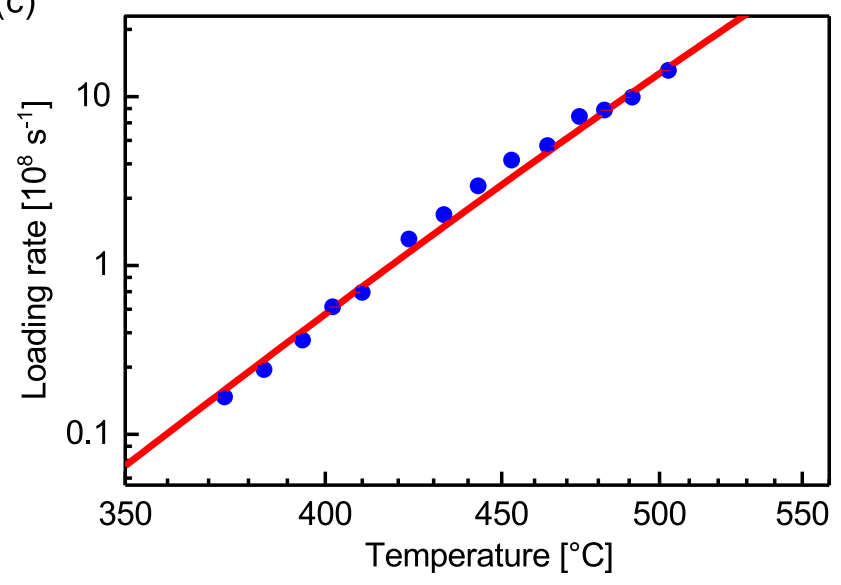

FIG. 4. Three-dimensional-magneto-optical-trap (3D-MOT) loading rate as a function of the pushing beam light intensity, the two-dimensional- (2D-) MOT cooling beam light intensity, and the oven temperature. For each panel, the unvaried parameters are set to $0.2 I_{\text {sat }}, 4 I_{\text {sat }}$ (four beams combined), and $465^{\circ} \mathrm{C}$, respectively. (a) Loading rate versus the pushing beam light intensity. The triangles indicate results of a model calculation. (b) Loading rate versus the 2D-MOT cooling beam light intensity. The solid line represents a fit with a model. (c) Loading rate versus the oven temperature (using flux enhancement of the 2D-MOT by the decreasing field Zeeman slower beam for this set of measurements). The solid line represents a model calculating the flux from the strontium vapor pressure and the 2D-MOT capture efficiency. Details on all model calculations can be found in the text. 